nehmen. Andere Refenrent ${ }^{\star}$ innen schlugen in diesem Zusammenhang auch vor, Care-Arbeit als über den Reproduktionsbereich hinausgehende vernetzende Praktiken zu denken.

Leider blieben viele Beiträge der Konferenz allerdings in Konzepten von Zweigeschlechtlichkeit verhaftet. Ein genauere Untersuchung von queeren Care-Praxen hätte weitere Ambivalenzen von Care, Gender und $\mathrm{Mi}$ gration sichtbar machen können.

\section{Care - mehr als ein Arbeits- verhältnis}

In vielen Beiträgen wurde Care als Arbeitsverhältnis diskutiert. Hierbei wurden Widersprüche zwischen informeller und professionalisierter Care-Arbeit deutlich. Wie können gleichzeitig sichere und faire Arbeitsbedingungen für Care-Arbeiter ${ }^{\star}$ innen und die Versorgung aller Menschen, die Unterstützung benötigen, organisiert werden, lautete eine wiederkehrende Frage.
Care-Arbeitsverhältnisse sind eingebunden in Netzwerke von Care und angebunden an Kämpfe um Aufenthalt und Arbeitsrecht. Um solche Schnittstellen von Kämpfen um reproduktive und sexuelle Rechte, Antirassismus und Ent_Hinderung ging es in einem projektübergreifenden Dialog, in dem Studierende vom Institut für Europäische Ethnologie der HU Berlin Arbeitsergebnisse präsentierten und dabei auch nach den Möglichkeiten und Grenzen des Care-Begriffs fragten.

Am Ende richteten die Teilnehme$r \star$ innen den Blick selbstkritisch auf die Tagung: Wie wurde mit Status-Unterschieden umgegangen? Feministische Wissensproduktionen zu Migration, Gender und Care enthalten zwangsläufig auch Ambivalenzen und Machtdynamiken. So fragten wir uns beispielsweise, welche Care-Arbeiter ${ }^{\star}$ innen während der Konferenz unsichtbar geblieben sind. Wer reinigt die Toiletten und Tagungsräume und unter welchen Arbeitsbedingungen findet eigentlich diese Reproduktionsarbeit statt?

\title{
Susanne Lettow
}

\section{Contested Kinship. Towards a Redefinition of Human Relations \\ Internationale Konferenz, Georg-August-Universität Göttingen, 14. -16. März 2019}

In den westlichen Gesellschaften herrschte lange Zeit ein Verständnis von Verwandtschaft vor, demzufolge verwandtschaftliche Zugehörigkeit primär als Blutsverwandtschaft verstanden wurde. Ein derart naturalisierendes Verständnis wurde in den verschiedenen Rechtssystemen privilegiert und erhält in der Gegenwart im Kontext der Genetik und der Nutzung genetischer Testverfahren zur Bestimmung von Verwandtschaftsverhältnissen erneut Bedeutung. 
Aus der Perspektive der Geschlechterforschung, der Sozial- und Kulturanthropologie sowie der Literatur- und Kulturwissenschaften sind aber spätestens seit den 1970er Jahren Zweifel an der Angemessenheit einer solchen naturalistisch-essentialistischen Konzeption angemeldet worden. Judith Butlers Essay über Antigone und Donna Haraways Plädoyer für ein erweitertes Verständnis von Verwandtschaft, das auch die Beziehungen zur nicht-menschlichen Welt umfasst, haben auf unterschiedliche Art und Weise konzeptionelle und politisch-ethische Alternativen zu den dominanten, in der Moderne am Modell der heterosexuell strukturierten Kleinfamilie orientierten, Verwandtschaftsvorstellungen skizziert. Die Tagung, die am Göttinger Centrum für Geschlechterforschung von Barbara Schaff, Inge Kroppenberg und Nikolaus Lindner organisiert wurde, knüpfte an diese Entwicklungen an. Ziel war es, durch eine breit angelegte interdisziplinäre Diskussion zur Etablierung und Weiterentwicklung des relativ jungen Feldes der Critical Kinship Studies beizutragen. Dabei lagen inhaltliche Schwerpunkte auf der Auseinandersetzung mit transnationalen Adoptionen, queeren Konstellationen von Verwandtschaft, den Verflechtungen von Verwandtschaft, Politik und Gewalt sowie die Frage nach der Bedeutung posthumanistischer Perspektiven für die kritische Analyse und Redefinition von Verwandtschaft. Quer zu den beteiligten Disziplinen - u.a. Anthropologie, Literaturwissenschaft, Rechtswissenschaft, Politikwissenschaft, Medizinethik, Geschichte und Philosophie - spielten zum einen Fragen der Temporalität von Verwandtschaft (etwa die Suche nach
Ursprüngen, imaginierte Genealogien, Entwürfe von Zukünftigkeit etc.) eine zentrale Rolle. Zum anderen wurde die Bedeutung von Narrativität für die Aushandlung von Verwandtschaftsbeziehungen aus unterschiedlichen Perspektiven beleuchtet. Margaret Homans rekonstruierte in ihrem Keynote Vortrag filmische Inszenierungen der Wiederbegegnung von durch Adoption getrennten Geschwistern. Die Auseinandersetzung mit Vergangenheit erhalte dabei, so Homans, nicht in erster Linie durch die Suche nach den verlorenen Eltern Bedeutung, sondern mit Blick auf die Aufdeckung der oft verschwiegenen Gründe der Trennungs- und Adoptionsgeschichten. In einem weiteren Keynote Vortrag skizzierte Elizabeth Peel das von ihr mitbegründete Feld der Critical Kinship Studies und markierte zentrale Felder zukünftiger Forschung. Dazu gehören Peel zufolge queere Verwandtschaftsbeziehungen, intergenerationelle Sorgeverhältnisse sowie die posthumanistische Erweiterung der Konzeption von Verwandtschaft durch die kritische Rekonstruktion von Praktiken der Grenzziehung zwischen Menschlichem und Nichtmenschlichem.

Insgesamt umfasste die Tagung acht Panels. Im ersten Panel ging es um Begriffe und Theorien der Verwandtschaft. In Anschluss an Butlers These, dass die Figur der Antigone nicht - wie bei Hegel - die Blutsverwandtschaft repräsentiert, sondern gerade für die historische Kontingenz von Verwandtschaftsnormen steht, wurde diskutiert, inwieweit ein historisierender Zugang, der auch Hegels Konstruktion als Auseinandersetzung mit einer historischspezifischen und intersektionalen »Krise der Verwandtschaft« begreift, Impul- 
se für eine kritische Analyse der Gegenwart liefert. Mit Blick auf die Gegenwart wurde problematisiert, dass auch die queere Neuverhandlung von Verwandtschaft oft durch traditionelle Vorstellungen von Partnerschaft und Familie geprägt ist, während radikalere Redefinitionen von Verwandtschaft marginalisiert werden. Das zweite Panel beschäftigte sich mit Prozessen der transnationalen Adoption. Silke Hackenesch zeigte, wie in den 1940er und 50er Jahren in den USA transnationale Adoptionen einerseits politisch codiert wurden, sofern es um die "Rettung" von Kindern aus faschistischen, postfaschistischen oder kommunistischen Ländern ging. Andererseits wurden durch die Adoption Schwarzer Kinder durch Weiße Eltern aber auch naturalisierende Vorstellungen von Familie in den USA unterminiert. Darüber hianus widmeten sich mehrere Beiträge den Perspektiven von Adoptierten, die lange Zeit weder in der Öffentlichkeit noch in der Forschung präsent waren. Dabei ging es anhand der Analyse aktueller Filme und literarischer Bearbeitungen des Themas sowie am Beispiel der Suche von Adoptierten aus Spanien nach ihren nepalesischen Herkunftsfamilien um die Frage, wie transnationale Verwandtschaftsverhältnisse hergestellt und ausgehandelt werden. Posthumanistische Zugänge zum Thema Verwandtschaft standen im Zentrum des dritten Panels. Mit Bezug auf Musikvideos von Björk, Donna Haraways Figuren von Cyborg und Göttin sowie Erzählungen von Olga Tokarczuk wurde die Frage aufgeworfen, welche Bedeutung Praktiken des "Sich-verwandtmachens" (Haraway) mit nichtmenschlichen Lebewesen und Artefakten für die Analyse und für Politiken der Geschlechterverhältnisse in der Gegenwart haben. Auch das vierte Panel setzte sich mit transnationalen Verwandtschaftsverhältnissen auseinander. Hier ging es zentral um Praktiken des rekinning, d.h. der Herstellung von Verwandtschaftsbeziehungen mit Herkunftsfamilien. Thematisiert wurden der Umgang mit struktureller Ungleichheit und kulturellen Differenzen am Beispiel von europäischen Adoptierten und Familien in Bolivien und mit Blick auf jene in den USA adoptierten Schwarzen Kinder, die seit den 1980er Jahren zunehmend Verbindung zu ihren Weißen deutschen Herkunftsfamilien aufnehmen und gleichzeitig an der multikulturellen Community Schwarzer Deutscher partizipieren. Deutlich wurde, dass im Prozess des rekinning sowohl soziale Beziehungen als auch die eigene Identität ausgehandelt werden. Maria Pia Guerra rekonstruierte anschließend das Spannungsverhältnis von Verwandtschaft, Nation und Migrationspolitik anhand der rechtlichen Regulierungen in Brasilien. Sie zeigte, wie der verfassungsrechtlich garantierte Schutz vor Ausweisung in den Perioden der Demokratie und der Diktatur unterschiedlich interpretiert wurde. Im Zentrum des fünften Panels stand das Verhältnis von biologischen und sozialen Dimensionen. Silke Schicktanz diskutiere die Frage, inwiefern im Verhältnis von Eltern und Kindern am Lebensende Sorgeverpflichtungen bestehen. Sie argumentierte, dass zu deren Begründung weder der Rekurs auf biologische Verwandtschaftsbeziehungen noch das moralphilosophische Konzept der Reziprozität ausreicht, da letzteres die vergeschlecht- 
lichten Sorge- und Machtverhältnisse außer Acht lässt. Zudem wurden naturalisierende Konzeptionen von Generativität und Verwandtschaft, die in der Reproduktionsindustrie vielfach aufgerufen werden, kritisiert. Das Verhältnis von Gewalt, Politik und Verwandtschaft wurde im sechsten Panel von Ashita Mandakathingal und Dragana Pejović thematisiert. Mandakathingal analysierte, welche Bedeutung von naturalisierten Vorstellungen von Verwandtschaft für die Ausübung staatlicher sexualisierter Gewalt, insbesondere Vergewaltigungen von Frauen der AdivasiGemeinschaften, in Indien haben. Pejović hingegen sprach über die Schwierigkeiten, das Gesetz gegen häusliche Gewalt umzusetzen, das 2016 in Serbien verabschiedet wurde. Der Gegensatz von Staat und Verwandtschaft, so Pejović, wird immer wieder angeführt um eine staatliche Intervention in familiäre Verhältnisse zurückzuweisen. Das siebente Panel befasste sich mit künstlerischen und literarischen Perspektiven, die zur kritischen De- und Rekonstruktion von Verwandtschaftsverhältnissen beitragen. Dabei ging es um Theaterstücke von Jean-Luc Lagarce und Bernard-Marie Koltès, in denen die Figur des Vaters destabilisiert und eine Neuordnung von Familienverhältnissen inszeniert wird; um Schwarze britische Gegenwartsliteratur, in der die Suche nach familiären und kulturellen Ursprüngen bearbeitet wird, sowie um die Schriften von Virgina Woolf und Vita Sackville-West. Kirsten Sandrock argumentierte, dass in der modernen Literatur genealogisch-lineare Vorstellungen inhaltlich sowie auf der Ebene der literarischen Form kritisiert werden. Im anschließenden achten $\mathrm{Pa}-$ nel wurde noch einmal der historische und geographische Horizont der Diskussion erweitert. Regina Schäfer diskutierte anhand von mittelalterlichen Quellen das Konzept der frunde, dessen Bedeutung sich sowohl vom modernen Begriff der Familie als auch dem der Freunde unterscheidet. Lovitoli Jimo analysierte mit Blick auf die Sumi Naga in Nordinidien, wie kulturelle Identität und Zugehörigkeit durch Praktiken der Eheschließung ausgehandelt werden.

Insgesamt erwies sich die Vielfalt der historischen, geographischen und methodischen Zugänge zur Frage, wie Verwandtschaft jenseits naturalisierender und essentialistischer Konzeptionen redefiniert werden kann, als äußerst fruchtbar. Für die weitere Forschung im Bereich der Critical Kinship Studies lieferte die Tagung eine Reihe wichtiger Impulse. Eine Frage, die es weiter und mit Blick auf die unterschiedlichen politischen, rechtlichen, ökonomischen und kulturellen Dimensionen von Verwandtschaft zu bearbeiten gilt, ist, wie abschließend festgehalten wurde, die Frage, wie Logiken der Ähnlichkeit und des Identischseins konstituiert werden und Zugehörigkeit begründen. 